\title{
Use of Two C Arm in Hip Fracture Surgery "The Sooner, The Better"
}

\section{Kashif Abbas $^{1^{*}}$ and Alexander Schuh ${ }^{2}$}

${ }^{1}$ Department of Orthopedic Surgery, Southampton University Hospitals NHS Trust, Pakistan

${ }^{2}$ Department of Orthopedic Surgery, Klinikum Neumarkt, Germany

Keywords: Traditionally intertrochanteric (IT) fractures

\section{Introduction}

Traditionally intertrochanteric (IT) fractures are being managed with extra medullary fixation devices. Recently intramedullary nailing has been successfully introduced for stable and unstable IT fracture. Use of intramedullary nail requires visualisation in two dimensions

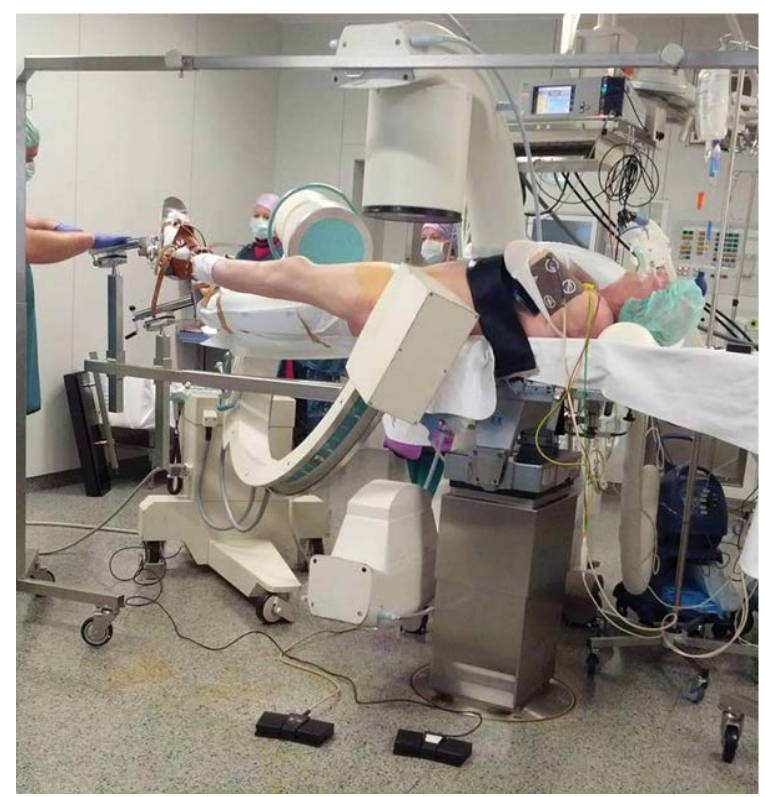

Figure 1

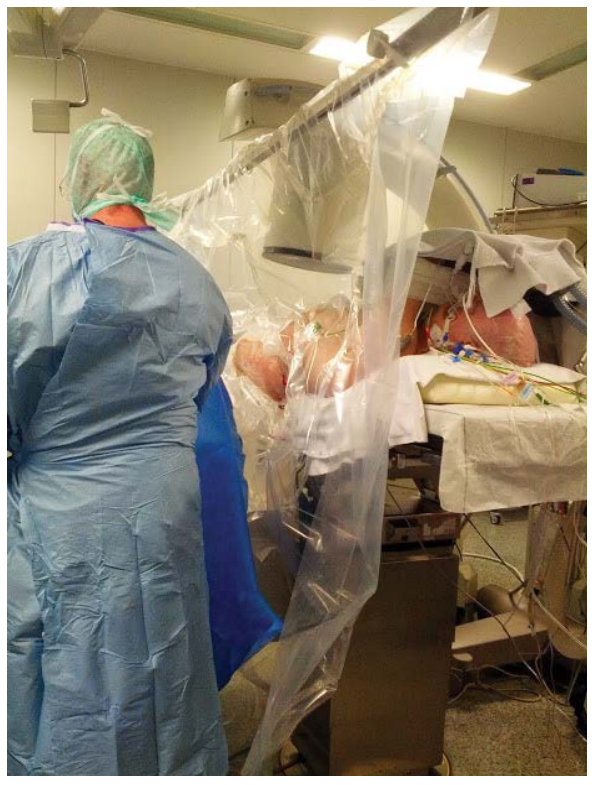

Figure 2 for correct portal of entry into the canal. Back and forth movement of the $\mathrm{C}$ arm in anteroposterior (AP) and lateral position is sometimes associated with loss of correct localization of the insertion point in eitherplane. To overcome this we have made a practice of using two $\mathrm{C}$ arm, positioning them in one plane each (Figure 1) before incision. Surgeon than stands at the top end and work through the gap between $\mathrm{X}$-ray tube and patient, after draping the image intensifier tube with sterile drape (Figure 2). Entry point is then confirmed in both plane simultaneously (Figure 3) which is followed byproximal hand reaming and nail preparation and insertion (Figure 4).

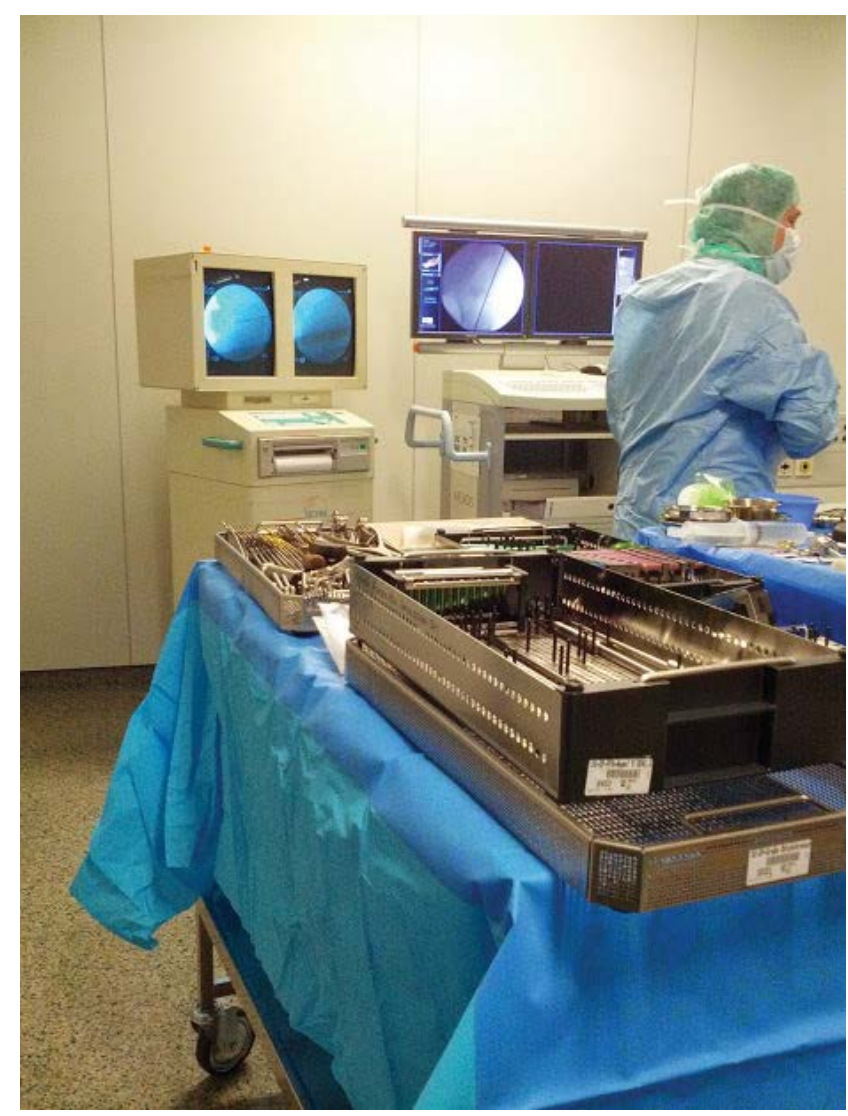

Figure 3

*Corresponding author: Kashif Abbas, Consultant orthopedic Surgeon, Pakistan, Tel: 00923002955041;E-mail: kashah_pk@yahoo.com

Received October 21, 2015; Accepted January 27, 2016; Published February 04, 2016

Citation: Abbas K, Schuh A (2016) Use of Two C Arm in Hip Fracture Surgery "The Sooner, The Better". Surgery Curr Res 6: i101. doi:10.4172/2161-1076.1000i101

Copyright: @ 2016 Abbas K, et al. This is an open-access article distributed under the terms of the Creative Commons Attribution License, which permits unrestricted use, distribution, and reproduction in any medium, provided the original author and source are credited. 


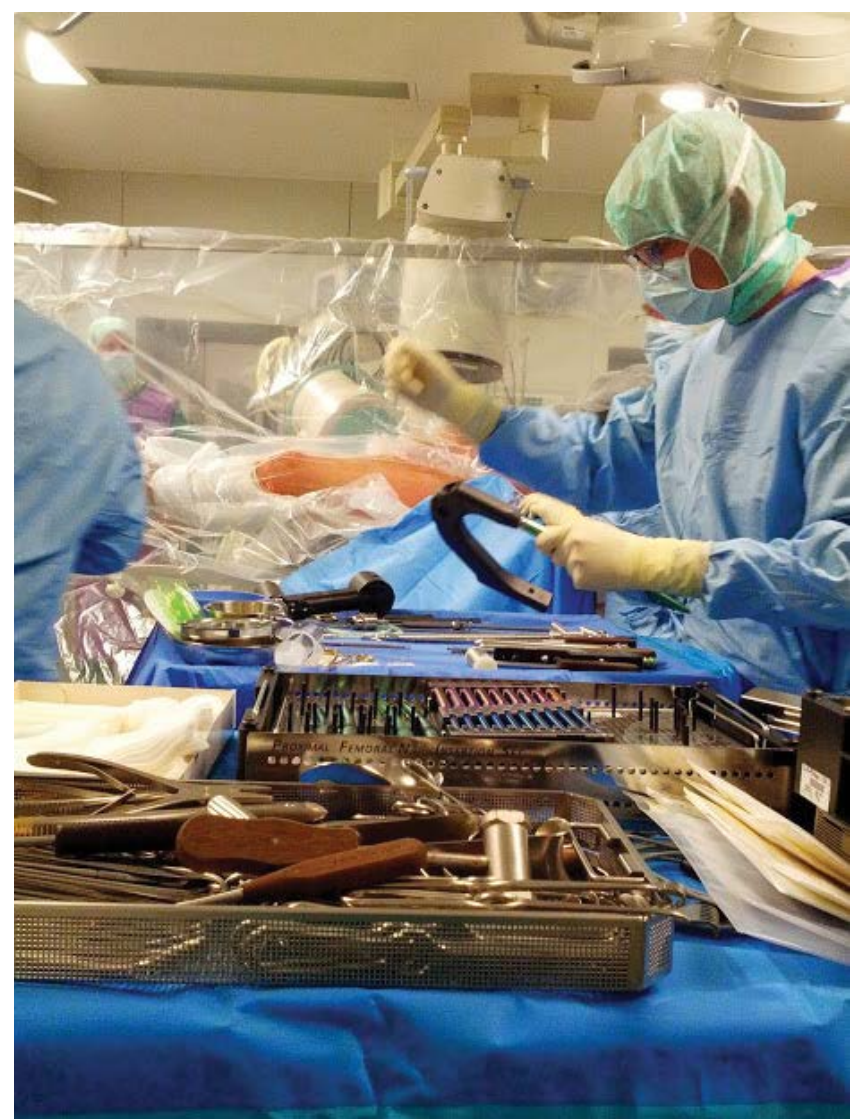

Figure 4

The adoption of this method has turned out to be very effective in reducing overall surgical timeand efforts. In our setting average time from incision to closure is 20 minutes. The only drawback is a requirement of additional $\mathrm{C}$ arm in a theatre and working through narrow window between fluoroscope tube (lateral plane) and patient. 